\title{
Design and Implementation of a Quadrifilar Helix Antenna Operating at 0.94GHz
}

\author{
Fang Qingyuan ${ }^{1}$, Song Lizhong ${ }^{2}$, Jin Ming ${ }^{2}$ and Qiao Xiaolin ${ }^{2}$ \\ ${ }^{1}$ School of Electronics Information on Engineering, \\ Harbin Institute of Technology, 150001, China \\ ${ }^{2}$ Harbin Institute of Technology at Weihai, \\ Harbin Institute of Technology,264209, China \\ fangqingyuanHIT@gmail.com,slz1975@sohu.com
}

\begin{abstract}
A quadrifilar helix antenna (QHA) operating at $0.94 \mathrm{GHz}$ is designed and realized in this paper. The designed QHA consists of four helical conductors which are excited in phase quadrature by employing a Wilkinson divider which shows good performance in return loss and transmission characteristic. According to the designed parameters of the helices and Wilkinson divider, a QHA is fabricated and measured. The measured results demonstrate that the designed QHA has a resonant frequency of $0.94 \mathrm{GHz}$ and relative good performances including low voltage standing wave ratio (VSWR), broad beam width and approximate circular polarization are achieved. The designed QHA has simple structure and is suitable for practical application.
\end{abstract}

Keywords: Quadrifilar helix antenna; Wilkinson divider; circular polarization; broad beam width

\section{Introduction}

The QHA has a lot of advantages such as compact structure, low cost, small near field radiation, wide cardioid pattern and so on [1-2]. The QHA was first proposed and researched by Kilgus [3-5]. After that, researches on the QHA for different applications are carried out for a long time. For example, in [6] a simple and innovative method for designing a spiral folded printed quadrifilar helix antenna (S-FPQHA) for dual-band operations is presented, and the axial length of a conventional PQHA is miniaturized approximately $43 \%$ by meandering and transforming the helix arms into the form of square spirals. In [7], a hollow ceramic rod is used as a dielectric load to reduce the size of QHA size, whose size is only $2.7 \%$ of an air-loaded QHA. In [8], a new folded printed quadrifilar helical antenna (FPQHA) is designed to achieve $30 \%$ of bandwidth in axial-mode. In [9], using meandering line techniques, the axial length of the conventional PQHA is reduced by 33\%. The traditional QHA has two resonant bifilar helix antennas oriented in a mutually orthogonal direction, which are fed by balanced fed structures. When the coaxial transmission line is used to feed the QHA, a balanced-to-unbalanced structure (Balun) is usually required. Generally the slot Balun, grounded coplanar waveguide $(\mathrm{GCPW})$ infinite feeding Baluns and natural infinite Balun are adopted. At the same time, a 90 degree phase difference between two bifilar helices is required to obtain the circular polarization radiation. The general methods to obtain this phase difference are two port quadrature feeding and self-phased technique. In this paper, a QHA fed by a Wilkinson divider operating at $0.94 \mathrm{GHz}$ is designed and implemented. The 
relative good performances including VSWR, beam width and polarization are observed according to the measured results, which validate the design of QHA in this paper.

\section{Quadrifilar Helix Antenna Structure}

The designed QHA consists of two bifilar helices and is fed by a divider. For each bifilar helix, an infinite Balun is used as the feed structure. A coaxial cable with diameter $\phi=1.2 \mathrm{~mm}$ is used as an arm of the bifilar helix. The inner conductor of the coaxial cable at the top of the helix is soldered to a wire conducting which is used as the other arm of the bifilar helix. The radii and heights of the helices are determined as $30 \mathrm{~mm}$ and $70 \mathrm{~mm}$, respectively. The turn number of the designed QHA is 0.5. Two uniform bifilar helices are orthogonally assembled in space to form the QHA. A divider is deigned to provide two exciting signals for these two bifilar helices. The amplitudes of these two signals are uniform. However, the phase difference of 90 degree exists between them. The geometry of the designed divider is shown in Figure 1.

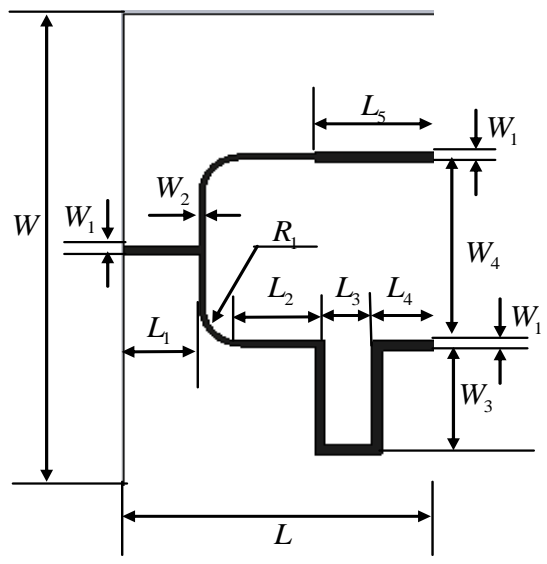

Figure 1. Geometry of the Power Divider

Here we still call this divider Wilkinson divider, although the is olation resistor is not used, which is usually contained in the traditional Wilkinson divider. The designed Wilkinson divider was fabricated through printed circuit technique. The thickness and relative permittivity of the dielectric substrate are $1 \mathrm{~mm}$ and 2.65 , respectively. The thick 3 ness of copper foil on the substrate is $0.036 \mathrm{~mm}$. In order to obtain the 90 degree phase difference between two output ports, we lengthened the microstrip transmission line of a port which is defined as the port 3. The output port with shorter transmission line is defined as the port 2. The input port is defined as the port 1 . The parameters of the Designed Wilkinson divider are determined by numerical simulations and the corresponding parameter values are as follows: $W=132.858 \mathrm{~mm}, \mathrm{~W} 1=2.682 \mathrm{~mm}$, $\mathrm{W} 2=1.488 \mathrm{~mm}, \quad \mathrm{~W} 3=29.203 \mathrm{~mm}, \quad \mathrm{~W} 4=50.597 \mathrm{~mm}, \quad \mathrm{R} 1=9.2562 \mathrm{~mm}, \quad \mathrm{~L}=81.550 \mathrm{~mm}$, $\mathrm{L} 1=20 \mathrm{~mm}, \quad \mathrm{~L} 2=22.147 \mathrm{~mm}, \quad \mathrm{~L} 3=12.318 \mathrm{~mm}, \quad \mathrm{~L} 4=16.341 \mathrm{~mm}, \quad \mathrm{~L} 5=\mathrm{L} 1+\mathrm{L} 4+\mathrm{W} 1$. The photograph of fabricated QHA is shown in Figure 2. 


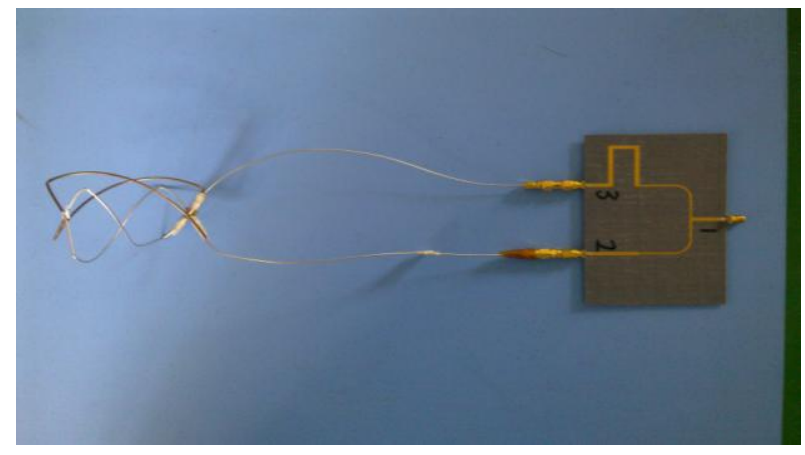

Figure 2. Photograph of the Fabricated QHA

\section{Measured Results}

The fabricated Wilkinson divider was measured by employing the Agilent vector network analyzer E8362B. Figure 3 shows the VSWR characteristics of three ports which are defined as in Figure 2. Acceptable impedance matching at port 1 can be observed from Figure 3. The measured transmission and isolation characteristics between three ports are shown in Figure 4.

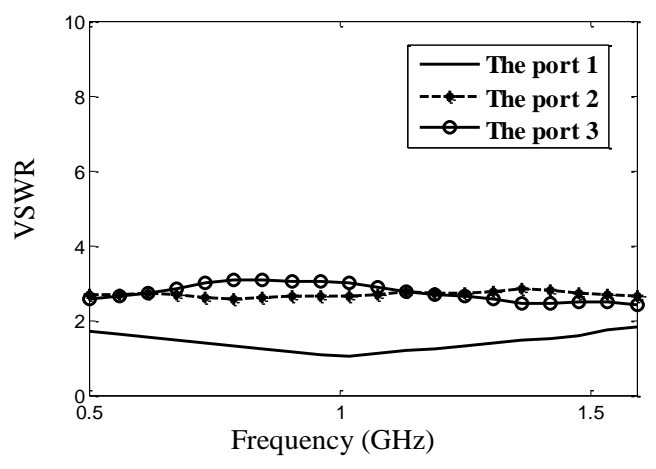

Figure 3. Measured VSWR of the Power Divider

It can be seen that the amplitude difference and phase difference between port 2 and port 3 are about $0.5 \mathrm{~dB}$ and 89 degree, respectively. It is obvious that the anticipated performances of the Wilkinson divider are realized. The Wilkinson divider and two bifilar helices are assembled into an entire QHA. Figure 6 shows the measured VSWR of the QHA.

It can be seen that the VSWR is less than 2 over the frequency range of $0.878 \mathrm{GHz}$ to $1.046 \mathrm{GHz}$ and the relative bandwidth is $17.87 \%$. It also can be observed that the VSWR is about 1.3 at $0.94 \mathrm{GHz}$. The radiation patterns on the azimuth and elevation plane at $0.94 \mathrm{GHz}$ of the QHA was measured in the anechoic chamber. Figure 6 shows the measured power radiation patterns. The beam widths on azimuth and elevation planes are about 135 degree and 116 degree, respectively. The front to back $(\mathrm{F} / \mathrm{B})$ ratio on azimuth plane is about $15 \mathrm{~dB}$ and it is about $10 \mathrm{~dB}$ in elevation plane. The small axial ratio (AR) is also observed according 
to the measured results, and the $\mathrm{AR}$ is about $0.0716 \mathrm{~dB}$ at the main radiation direction, which reveal the designed QHA has radiation characteristic of approximate circular polarization.

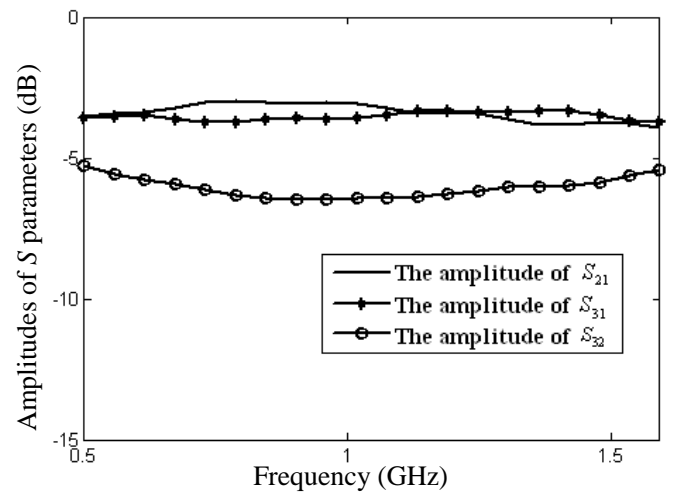

(a)

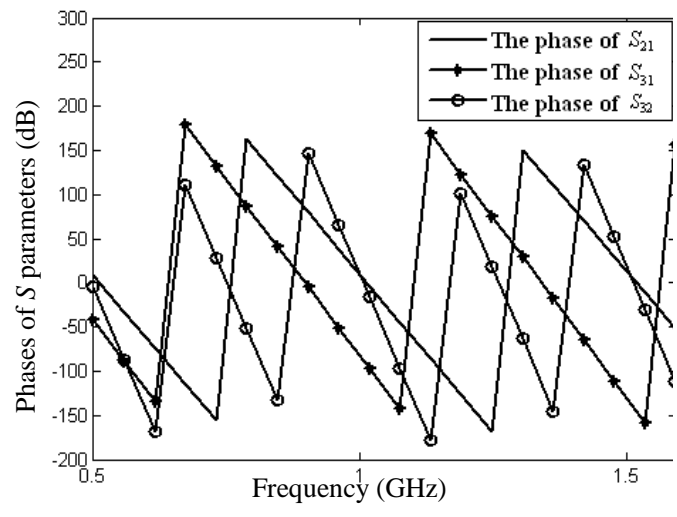

(b)

Figure 4. Measured Transmission and Isolation Characteristics of the Fabricated Power Divider: (a) the Amplitudes of S Parameters (b) the Phases of $S$ Parameters

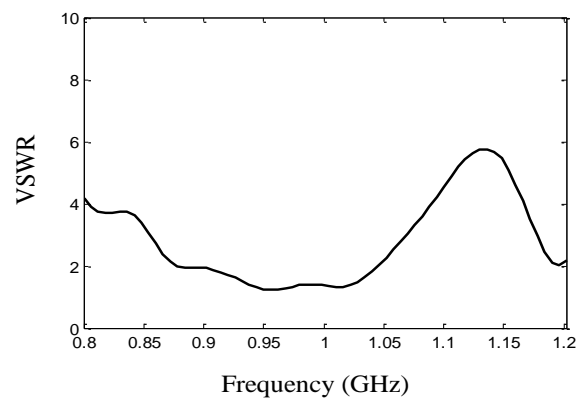

Figure 5. Measured VSWR of the Fabricated QHA

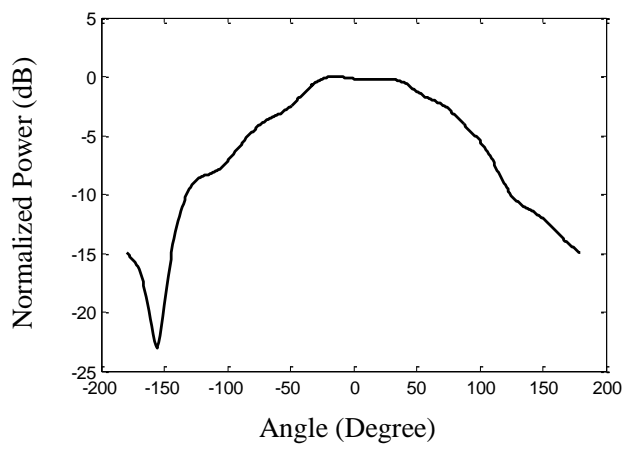

(a)

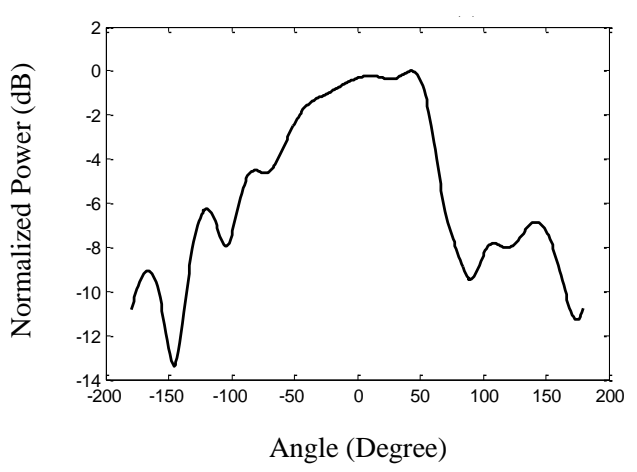

(b)

Figure 6. Measured Power Patters of the Fabricated QHA: (a) the Azimuth Plane (b) the Elevation Plane 


\section{Conclusion}

This paper has designed and fabricated a QHA operating at $0.94 \mathrm{GHz}$. A Wilkinson divider was also designed to realize the two port quadrature feed of the QHA. The experimental results of the QHA are provided which illustrate relative good performances of the designed QHA. The provided designed parameters of QHA in this paper are helpful to the design and implementation of QHA in practical engineering.

\section{Acknowledgements}

The authors would like to thank LIU Xinlei and Zhang Dequan for their help in antenna measurement in anechoic chamber.

\section{References}

[1] M. Amin and R. Cahill, "Bandwidth limitation of two-port fed and self-phased quadrifilar helix antennas", Microwave and Optical Technology Letters, vol. 46, no. 1, (2005).

[2] Y. Wai Chow, E. Kai Ning Yung and H. Tat Hui, "Quadrifilar helix antenna with parasitic helical strips", Microwave and Optical Technology Letters, vol. 30, no. 2, (2001).

[3] C. C. Kilgus, "Multielement fractional turn helices", IEEE Transactions on Antennas and Propagation, vol. 16 , no. $4,(\mathbf{1 9 6 8})$.

[4] C. C. Kilgus, "Resonant quadrifilar helix", IEEE Transactions on Antennas and Propagation", vol. 17, no. 3, (1969).

[5] C. C. Kilgus, "Shaped-conical radiation pattern performance of the backfire quadrifilar helix", IEEE Transactions on Antennas and Propagation, vol. 23, no. 3, (1975).

[6] J. Rabemanantsoa and A. Sharaiha, "Size Reduced Multi-Band Printed Quadrifilar Helical Antenna", IEEE Transactions on Antennas and Propagation, vol. 59, no. 9, (2011).

[7] Y.-S. Wang and S.-J. Chung, "A Miniature Quadrifilar Helix Antenna for Global Positioning Satellite Reception", IEEE Transactions on Antennas and Propagation, vol. 57, no. 12, (2009).

[8] Y. Letestu and A. Sharaiha, "Broadband Folded Printed Quadrifilar Helical Antenna", IEEE Transactions on Antennas and Propagation, vol. 54, no. 5, (2006).

[9] M. G. Ibambe, Y. Letestu and A. Sharaiha, "Compact printed quadrifilar helical antenna", Electronics Letters, vol. 43, no. 13, (2007). 
International Journal of Future Generation Communication and Networking Vol.6, No.6 (2013) 